\title{
ABC: Algebraic Bound Computation for Loops *
}

\author{
Régis Blanc ${ }^{1}$, Thomas A. Henzinger ${ }^{2}$, Thibaud Hottelier ${ }^{3}$, and Laura Kovács ${ }^{4}$ \\ 1 EPFL \\ 2 IST Austria \\ 3 UC Berkeley \\ 4 TU Vienna
}

\begin{abstract}
We present $\mathrm{ABC}$, a software tool for automatically computing symbolic upper bounds on the number of iterations of nested program loops. The system combines static analysis of programs with symbolic summation techniques to derive loop invariant relations between program variables. Iteration bounds are obtained from the inferred invariants, by replacing variables with bounds on their greatest values. We have successfully applied ABC to a large number of examples. The derived symbolic bounds express non-trivial polynomial relations over loop variables. We also report on results to automatically infer symbolic expressions over harmonic numbers as upper bounds on loop iteration counts.
\end{abstract}

\section{Introduction}

Establishing tight upper bounds on the execution times of programs is both difficult and interesting, see e.g. $[10,5,9,8]$. We present $\mathrm{ABC}$, a new software tool for automatically computing tight symbolic upper bounds on the number of iterations of nested program loops. The derived bounds express polynomial relations over loop variables. ABC is fully automatic, combines static analysis of programs with symbolic summation techniques, and requires no user-guidance in providing additional set of predicates, templates and assertions. ABC is also able to derive symbolic expressions over harmonic numbers as upper bounds on loop iteration counts, which, to the best of our knowledge, is not yet possible by other works.

In our approach to bound computation, we have identified a special class of nested loop programs, called the $A B C$-loops (Section 3.1). Further, we have built a loop converter to transform, whenever possible, arbitrary loops into their equivalent $\mathrm{ABC}$-loop format (Section 3.2). Informally, an ABC-loop is a nested for-loop such that each loop from the nested loop contains exactly one iteration variable with only one condition and one (non-initializing) update on the iteration variable. For such loops, our method derives precise bounds on the number of loop iterations.

In our work, we assume that each program statement is annotated with the time units it needs to be executed. For simplicity, we assume that an iteration of an unnested loop takes one unit time, and all other instructions of the unnested loop need zero time.

The key steps of our approach to bound computation are as follows (Section 3.3). (i) First, we instrument the innermost loop body of an ABC-loop with a new variable

\footnotetext{
* This work was supported in part by the Swiss NSF. The fourth author is supported by an FWF Hertha Firnberg Research grant (T425-N23).
} 
that increases at every iteration of the program. We denote this variable by $z$. Upper bounds on the value of $z$ thus express upper bounds on the number of loop iterations. (ii) Next, the value of $z$ is computed as a polynomial function over the nested loop's iteration variables. We call the relation between $z$ and the loop's iteration variables the $z$-relation. To this end, for each loop of the ABC-loop, recurrence equations of $z$ and the loop iteration variables are first constructed. Closed forms of variables are then derived using our symbolic solver which integrates special techniques from symbolic summation (Section 3.4). The derived closed forms express valid relations between $z$ and the loop iteration variables, and thus the $z$-relations are loop invariant properties. (iii) Further, by replacing loop iteration variables by bounds on their greatest values in the computed $z$-relation, bounds on the value of $z$ are obtained. These bounds give us tight symbolic upper bounds on the number of iterations of the program. Our method can be generalized for the timing analysis of loops whose iteration bounds involve harmonic expressions over the loop variables (Section 3.5).

Implementation. ABC was implemented in the the Scala programming language [18], contains altogether 5437 lines of Scala code, and is available at:

http://mtc.epfl.ch/software-tools/ABC/

Inputs to $\mathrm{ABC}$ are loops written in the Scala syntax. $\mathrm{ABC}$ first rewrites the input loop into an equivalent $\mathrm{ABC}$-loop by using its loop converter, and then computes bounds on loop iteration counts using its bound computer. The bound computer relies on the symbolic solver in order to derive closed forms of symbolic sums and simplify mathematical expressions. The overall workflow of $\mathrm{ABC}$ is given in Figure 1.

Note that $A B C$ does not rely on an external computer algebra package for symbolic summation.

Experiments. We successfully applied ABC on examples from [10,9], as well as on 90 nested loops extracted from the JAMA package [13] - see Section 4 and the mentioned $\mathrm{URL}^{5}$. Altogether, we ran ABC on 558 lines of JAMA. ABC computed precise upper bounds on iteration counts for all loops, and inferred the $z$-relation for 87 loops, all in less than one second on a machine with a $2.8 \mathrm{GHz}$ Intel Core 2 Duo processor and $2 \mathrm{~GB}$ of RAM. The 3 loops for which ABC was not able to derive the $z$-relation were actually sequences of loops.

We believe that similar experimental results as the ones resulting from JAMA could be obtained by running $\mathrm{ABC}$ on the Jampack library [20], or on various numerical packages of computer algebra packages such as Mathematica [22], Matlab [4], or Mathcad [2].

Related work. We only discuss some of the many methods that are related to ABC.

Paper [15] infers polynomial loop invariants among program variables by using polynomial invariant templates of bounded degree. Unlike [15], where no restrictions on the considered loops were made, we require no user guidance in providing invariant

\footnotetext{
${ }^{5}$ There are 167 loops in JAMA amongst which there are 90 nested for-loops. ABC successfully
} inferred the exact bound for all but three for-loops. 


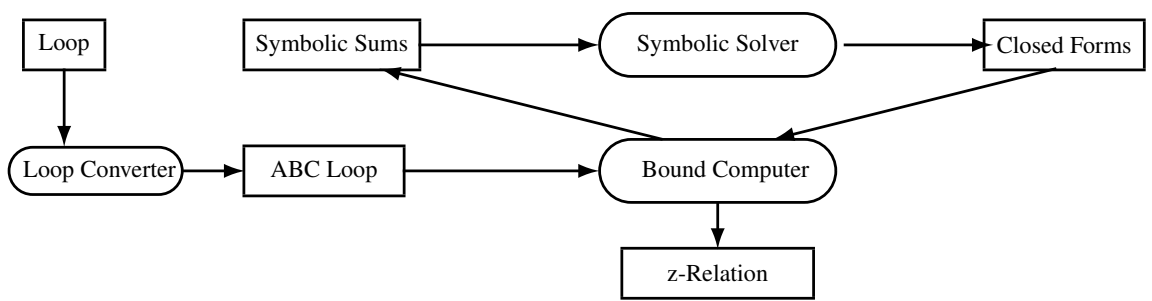

Fig. 1. The ABC tool.

templates but automatically derive invariants ( $z$-relations) for a restricted class of loops. Our method has thus advantage in automation, but it is restricted to ABC-loops.

The approach presented in [11] infers invariants and bound assertions for loops with nested conditionals and assignments, where the assignments statements describe non-trivial recurrence relations over program variables (i.e. variable initializations are not allowed). To this end, loops are first represented by a collection of loop-free program paths, where each path corresponds to one conditional branch. Further, recurrence solving over variables is applied on each program path separately. Bounds on iteration counters can be finally inferred if the iteration counters are changed by each path in the same manner. Due to these restrictions, nested loops cannot be handled in [11]. Contrarily to [11], we infer bound assertions as $z$-relations for nested loops, but, unlike [11], our invariant assertions are only over loop iteration variables and not arbitrary program variables.

Paper [10] derives iteration bounds of nested loops by pattern matching simple recurrence equations. Contrarily to [10], we solve more general recurrence equations using the Gosper algorithm [6] and identities over harmonic numbers [7].

Solving recurrence relations is also the key ingredient in [1] for computing bounds. Unlike our method, evaluation trees for the unfoldings of the recurrence relations are first built in [1], and closed forms of recurrences are then derived from the maximum size of the trees. Contrarily to [1], we can handle more general recurrences by means of symbolic computation, but [1] has the advantage of solving non-deterministic recurrences that may result from the presence of guards in the loop body.

Symbolic upper bounds on iteration counts of multi-path loops are automatically derived in [8]. The approach deploys control-flow refinement methods to eliminate infeasible loop paths and rewrites multi-path loops into a collection of simpler loops for which bound assertions are inferred using abstract interpretation techniques [3]. The programs handled by [8] are more general than the ABC-loops. Unlike [8], we do not rely on abstract interpretation, and are able to infer harmonic expressions as upper bounds on loop iterations counts. Abstract interpretation is also used in $[12,16]$ for automatically inferring upper and lower bounds on the number of execution steps of logic programs.

Paper [14] describes an automated approach for inferring linear upper bounds for functional programs, by solving constraints that are generated using linear programming. In our work we derive polynomial, and not just linear, upper bounds. 


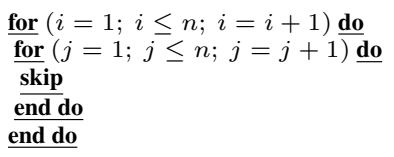

(a)

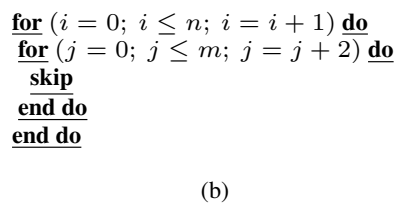

Fig. 2. Examples illustrating the power of $\mathrm{ABC}$ to (i) compute $z$-relations as loop invariants, and (ii) infer tight upper bounds on the number of iterations of loops.

There has been a great deal of research on estimating the worst case execution time (WCET) of real-time systems, see e.g. [5, 9, 19]. Papers [5,9] automatically infer loop bounds only for simple loops; bounds for the iteration numbers of multi-path loops must be provided as user annotations. The aiT tool [5] determines the number of loop iterations by relying on a combination of interval-based abstract interpretation with pattern matching on typical loop patterns. The SWEET tool [9] determines upper bounds on loop iterations by unrolling loops dynamically and analyzing each loop iteration separately using abstract interpretation. In contrast, our method is fully automatic and path-insensitive, but it is restricted to ABC-loops. The TuBound tool [19] implements a constraint logic based approach for loop analysis to compute tight bounds for nestedloops. The constraints being solved in [19] are directly obtained from the loop conditions and express bounds on the loop iteration variables. Unlike [19], we infer loop bounds by computing closed forms of iteration variables.

\section{Motivating Examples}

We first give some examples illustrating what kind of iteration bounds $\mathrm{ABC}$ can automatically generate.

Consider Figure 2(a) taken from the JAMA library [13]. ABC first instruments the innermost loop of Figure 2(a) with a new variable $z$, initialized to 1, for counting the number of iterations of Figure 2(a). The thus obtained loop is presented in Figure 3(a). Further, by applying $\mathrm{ABC}$ on Figure 3(a), we derive the $z$-relation ${ }^{6}$ :

$$
z=(i-1) n+j
$$

as an invariant property of the loop. By replacing $i$ and $j$ with bounds on their greatest values (i.e. $n$ ) in the $z$-relation, the number of iterations of Figure 2(a) is bounded by:

$$
n^{2} \text {. }
$$

Consider next Figure 2(b) with a non-unit increment, and its "instrumented" version in Figure 3(b). We obtain the $z$-relation:

$$
z=1+\left\lfloor\frac{j}{2}\right\rfloor+i\left(\left\lfloor\frac{m}{2}\right\rfloor+1\right)
$$

\footnotetext{
${ }^{6}$ Actually, the loops of Figure 2 are first translated into their equivalent ABC-format, and then the $z$ variable is introduced in their innermost loop body. For simplicity, in Figure 3 we present the "instrumentation" step directly on the loops of Figure 2 and not on their ABC-loop formats.
} 


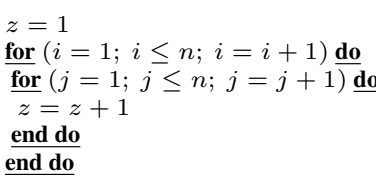

(a)

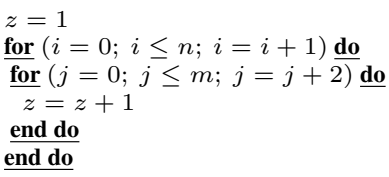

(b)

Fig. 3. Figure 2 instrumented by $\mathrm{ABC}$.

yielding:

$$
1+(1+n)\left\lfloor\frac{m}{2}\right\rfloor+n
$$

as a tight upper bound on loop iteration counts ${ }^{7}$, where $\left\lfloor\frac{m}{2}\right\rfloor$ denotes the largest integer not greater than $\frac{m}{2}$.

In the sequel, we illustrate the main steps of $\mathrm{ABC}$ on Figure 2(b).

\section{ABC: System Description}

We have identified a special class of loops, called the $A B C$-loops (Section 3.1), and designed a loop converter for translating programs into their equivalent $\mathrm{ABC}$-loop shape (Section 3.2). Algorithmic methods from symbolic summation, implemented in our symbolic solver (Section 3.4), are further deployed in $\mathrm{ABC}$ to automatically derive upper bounds on loop iterations of ABC-loops (Section 3.3).

\subsection{ABC-Loops}

We denote by $\mathbb{Z}$ the set of integer numbers, and by $\mathbb{Z}[x]$ the ring of polynomial functions in indeterminate $x$ over $\mathbb{Z}$.

We consider programs of the following form:

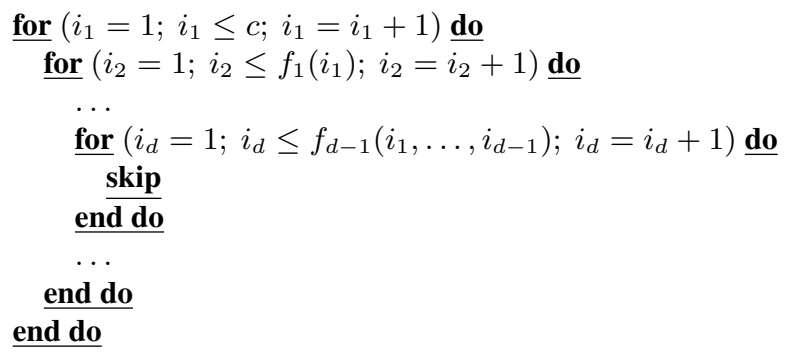

\footnotetext{
${ }^{7}$ In our work we did not consider analyzing the relations between the smallest and greatest symbolic values of the loop iteration variables. It may however be the case that these symbolic values are such that the loops are never executed (e.g. $n<0$ ).
} 


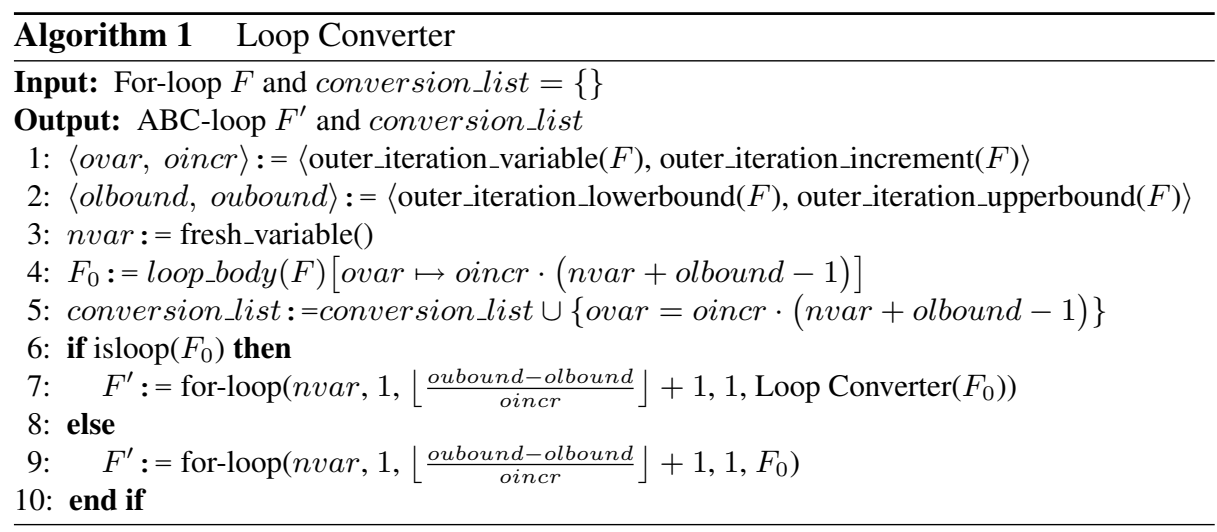

where $i_{1}, \ldots, i_{d}$ are pairwise disjoint scalar variables (called loop iteration variables) with values from $\mathbb{Z}, c$ is an integer-valued symbolic constants, and $f_{k} \in \mathbb{Z}\left[i_{1}, \ldots, i_{k}\right]$ are polynomial functions $(k=1, \ldots, d-1)$.

In what follows, loops satisfying (1) will be called $A B C$-loops.

\subsection{The Loop Converter}

Converting loops into ABC-loops is done as presented in Algorithm 1. The algorithm (i) converts loops into equivalent ones such that the smallest values of the loop iteration variables are 1 , and (ii) converts loops with arbitrary increments over the iteration variables into equivalent loops with increments of 1 . The for-loop $\left(\mathrm{v}, e_{1}, e_{2}, e_{3}\right.$, body) notation used in Algorithm 1 is a short-hand notation for the loop:

$$
\underline{\text { for }}\left(v=e_{1} ; v \leq e_{2} ; v=v+e_{3}\right) \underline{\text { do }} b o d y \text { end do. }
$$

In more detail, Algorithm 1 takes as input a nested for-loop $F$ and an empty list conversion_list, and returns, whenever possible, an ABC-loop $F^{\prime}$ that is equivalent to $F$. The conversion_list is used to store the list of changes made by Algorithm 1 on the iteration variables of $F$.

Lines 4-9 of Algorithm 1 are required to convert $F$ into an equivalent loop whose outermost loop has the following properties: it iterates over a new variable nvar instead of the iteration variable ovar of the outermost loop of $F$, where nvar and ovar are polynomially related; the smallest value of nvar is 1 (instead of the smallest value olbound of ovar); nvar is increment by 1 (instead of the oincr increment value of ovar); the greatest value of nvar is given by the largest integer not greater than the rational expression $\frac{\text { oubound-olbound }}{\text { oincr }}+1$, where oubound is the greatest value of ovar. The appropriately modified ${ }^{8}$ loop body $F_{0}$ of $F$ is processed in the similar manner, yielding finally the ABC-loop $F^{\prime}$ that is equivalent to $F$.

\footnotetext{
${ }^{8}$ The expression $s[x \mapsto e]$ denotes the expression obtained from $s$ by substituting each occurrence of the variable $x$ by the expression $e$.
} 


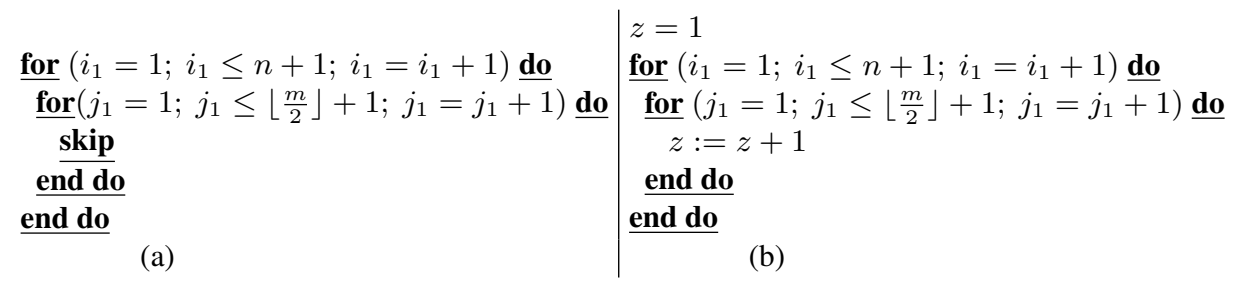

Fig. 4. ABC-loop format of Figure2(b) and its instrumented version, where $i=i_{1}-1$ and $j=2\left(j_{1}-1\right)$. Note that $\lfloor m / 2\rfloor \in \mathbb{Z}$.

Example 1. Consider Figure 2(b). By applying Algorithm 1, the loop iteration variables $i_{1}$ and $j_{1}$ are introduced with $i=i_{1}-1$ and $j=2\left(j_{1}-1\right)$ (lines 3-5 of Algorithm 1). The smallest values of $i_{1}$ and $j_{1}$ are 1 , their greatest values are respectively $n+1$ and $\left\lfloor\frac{m}{2}\right\rfloor+1$, and $i_{1}$ and $j_{1}$ are incremented by 1 (lines 6-9 of Algorithm 1). The ABC-loop format of Figure 2(b) is given in Figure 4(a).

Based on Algorithm 1 and keeping the notations of (1), we conclude that the general shape of loops that can be converted into ABC-loops is:

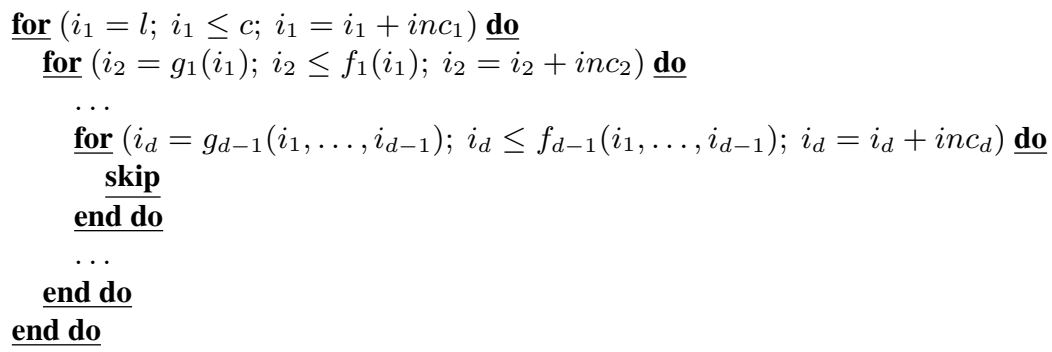

where $l, i n c_{1}, \ldots, i n c_{d}$ are integer-valued symbolic constants, and $g_{k} \in \mathbb{Z}\left[i_{1}, \ldots, i_{k}\right]$.

\subsection{The Bound Computer}

We assume that each program statement is annotated with the time units it needs to be executed. For simplicity, we assume that an iteration of an unnested ABC-loop takes one time unit, and all other instructions of the unnested loop need zero time (e.g. assignment statements take zero time to be executed). That is we compute a bound on the total number of loop iterations of an ABC-loop (1).

In our approach to bound computation, we instrument the innermost loop body of (1) with a new variable that increases at every iteration of the program, and is initialized to 1 before entering the ABC-loop. We denote this variable by $z$. From (1), we thus 
obtain:

$$
\begin{aligned}
& z=1 \\
& \text { for }\left(i_{1}=1 ; i_{1} \leq c ; i_{1}=i_{1}+1\right) \underline{\mathbf{d o}} \\
& \text {... } \\
& \text { for }\left(i_{d}=1 ; i_{d} \leq f_{d-1}\left(i_{1}, \ldots, i_{d-1}\right) ; i_{d}=i_{d}+1\right) \underline{\mathbf{d o}} \\
& z:=z+1 \\
& \text { end do } \\
& \text {... }
\end{aligned}
$$

Example 2. The instrumented loop of Figure 4(a) is given in Figure 4(b).

Upper bounds on the value of $z$ give upper bounds on the number of iterations of (3). We are hence left with computing the value of $z$ as a function, called the $z$-relation, over $i_{1}, \ldots, i_{d}$. To this end, the value of $z$ at an arbitrary iteration of the outermost loop of (3) is first computed.

Computing the value of $z$ after an arbitrary iteration of the outermost loop of (3). Let us consider a more general loop than (3):

$$
\begin{aligned}
& \underline{\text { for }}\left(i_{1}=1 ; i_{1} \leq c ; i_{1}=i_{1}+1\right) \underline{\mathbf{d o}} \\
& \quad \underline{\text { for }}\left(i_{2}=1 ; i_{2} \leq f_{1}\left(i_{1}\right) ; i_{2}=i_{2}+1\right) \underline{\text { do }} \\
& \quad \ldots \\
& \quad \underline{\text { for }}\left(i_{d}=1 ; i_{d} \leq f_{d-1}\left(i_{1}, \ldots, i_{d-1}\right) ; i_{d}=i_{d}+1\right) \underline{\text { do }} \\
& \quad \begin{array}{l}
z:=z+g\left(i_{d}\right) \\
\text { end do }
\end{array} \\
& \quad \begin{array}{l}
\text { end do } \\
\text { end do }
\end{array}
\end{aligned}
$$

where $i_{1}, \ldots, i_{d}, c, f_{1}, \ldots, f_{d-1}$ are as in (1), and $g \in \mathbb{Z}\left[i_{d}\right]$. In particular, if $g=1$ then (4) becomes (3).

Let $s_{1}, \ldots, s_{l}$ be nonnegative integers $(l=1, \ldots, d)$ such that $1 \leq s_{1} \leq c$, $1 \leq s_{2} \leq f_{1}\left(i_{1}\right), \ldots$, and $1 \leq s_{l} \leq f_{l-1}\left(i_{1}, \ldots, i_{l-1}\right)$. In the sequel we consider $s_{1}, \ldots, s_{l}$ arbitrary but fixed. We write $x^{\left(l,\left\|s_{1}, \ldots, s_{l}\right\|\right)}$ to mean the value of a variable $x \in\left\{i_{1}, \ldots, i_{d}, z\right\}$ in (4) such that the $k$ th loop of (4) is at its $s_{k}$ th iteration $(k=1, \ldots, l)$,

We are thus interested in deriving $z^{\left(1,\left\|s_{1}\right\|\right)}$ for $s_{1} \in\{1, \ldots, c\}$. We proceed as follows. For each loop of (4), starting from the innermost one, we (i) model the assignment over $z$ as a recurrence equation, (ii) deploy symbolic summation algorithms to compute the closed form of $z$, and (iii) replace the loop by a single assignment over $z$ expressing the relation between the values of $z$ before the first and after the last execution of the loop. Steps (i)-(iii) are recursively applied until all loops of (4) are eliminated.

In more detail, $z^{\left(1,\left\|s_{1}\right\|\right)}$ is derived as follows. We start with the innermost loop of (4). The assignment over $z$ is modeled by the recurrence equation:

$$
z^{\left(d,\left\|s_{1}, \ldots, s_{d}+1\right\|\right)}=z^{\left(d,\left\|s_{1}, \ldots, s_{d}\right\|\right)}+g\left(i_{d}^{\left(d,\left\|s_{1}, \ldots, s_{d}-1\right\|\right)}\right),
$$




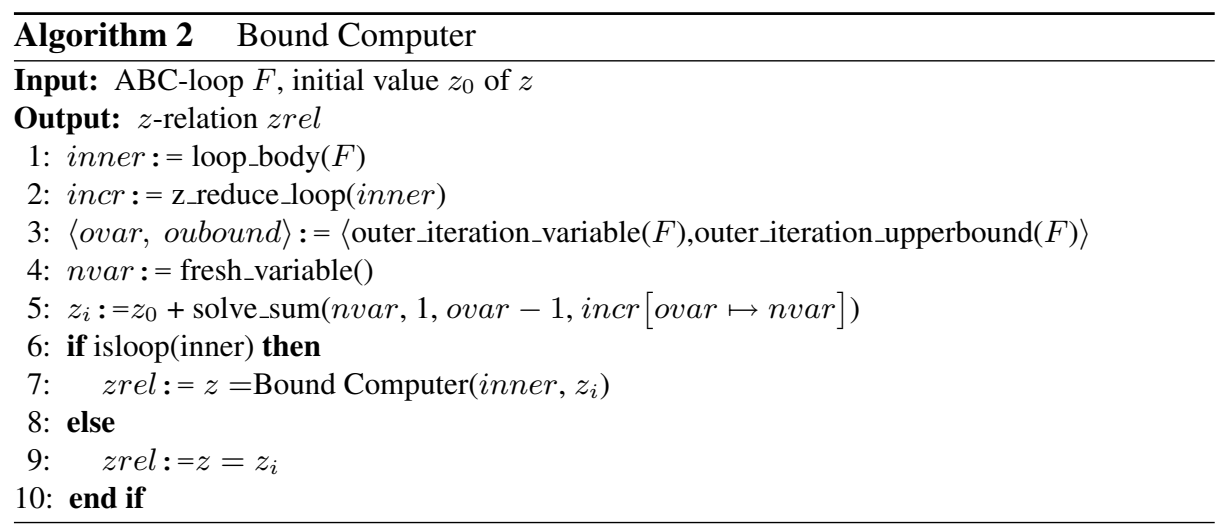

yielding:

$$
z^{\left(d,\left\|s_{1}, \ldots, s_{d}\right\|\right)}=i n i_{z}+\sum_{k=1}^{s_{d}} g\left(i_{d}^{\left(d,\left\|s_{1}, \ldots, k-1\right\|\right)}\right),
$$

where $i n i_{z}=z^{\left(d,\left\|s_{1}, \ldots, 0\right\|\right)}$ denotes the value of $z$ before entering the innermost loop of (4). The value of $i_{d}^{\left(d,\left\|s_{1}, \ldots, s_{d}\right\|\right)}$ is computed from the recurrence equation:

$$
i_{d}^{\left(d,\left\|s_{1}, \ldots, s_{d}+1\right\|\right)}=i_{d}^{\left(d,\left\|s_{1}, \ldots, s_{d}\right\|\right)}+1 .
$$

Namely, we have $i_{d}^{\left(d,\left\|s_{1}, \ldots, s_{d}\right\|\right)}=i n i_{d}+\sum_{k=1}^{s_{d}} 1$, where $i n i_{d}=1$ denotes the initial value of $i_{d}$ (i.e. before the first iteration of the innermost loop of (4)). Hence,

$$
i_{d}^{\left(d,\left\|s_{1}, \ldots, s_{d}\right\|\right)}=s_{d}+1
$$

Note that (6) holds for each iteration variable, that is:

$$
i_{l}^{\left(l,\left\|s_{1}, \ldots, s_{l}\right\|\right)}=s_{l}+1
$$

for every $l \in\{1, \ldots, d\}$. For this reason, in what follows we write $i_{l}$ instead of $i_{l}^{\left(l,\left\|s_{1}, \ldots, s_{l}\right\|\right)}$ and use the relation $i_{l}=s_{l}+1$ to speak about the value of $i_{l}$ at iteration $s_{l}$ of the $l$ th loop. We thus obtain:

$$
z^{\left(d,\left\|s_{1}, \ldots, s_{d}\right\|\right)}=i n i_{z}+\sum_{k=1}^{s_{d}} g\left(i_{d}^{\left(d,\left\|s_{1}, \ldots, k-1\right\|\right)}\right)=i n i_{z}+\sum_{k=1}^{i_{d}-1} g(k)
$$

Since $g \in \mathbb{Z}\left[i_{d}\right]$, the closed form of $\sum_{k=1}^{i_{d}-1} g(k)$ always exists [6] and can be computed as a polynomial function over $i_{d}$ (see Section 3.4).

Finally, we consider the last iteration $s_{d}=i_{d}-1=f_{d-1}\left(i_{1}, \ldots, i_{d-1}\right)$ of the innermost loop of (4), and write incr $_{d}=\sum_{k=1}^{f_{d-1}\left(i_{1}, \ldots, i_{d-1}\right)} g(k)$. We make use of incr $_{d} \in \mathbb{Z}\left[i_{1}, \ldots, i_{d-1}\right]$ to "eliminate" the innermost loop of (4) and obtain: 


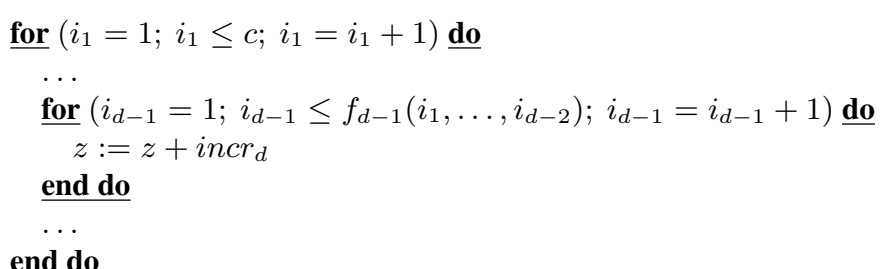

end do

Inner loops of (7) can be further eliminated by applying recursively the steps described above, since closed forms of polynomial expressions over $i_{1}, \ldots, i_{d}$ yield polynomial expressions over $i_{1}, \ldots, i_{d}$ whenever the summation variables are bounded by polynomial expressions. As a result, the total number of increments over $z$ in the $s_{1}$ th iteration of the outermost loop of (4) is derived. Let us denote this number by incr $_{1}$. Then:

$$
z^{\left(1,\left\|s_{1}\right\|\right)}=z_{0}+i n c r_{1} \text {, where } z_{0}=1 \text { is the value of } z \text { before (4). }
$$

Example 3. Consider Figure 4(b). We aim at deriving $z^{\left(1,\left\|s_{1}\right\|\right)}$, where $1 \leq s_{1} \leq n+1$ is arbitrary but fixed such that $i_{1}=s_{1}+1$.

From the innermost loop of Figure 4(b), we have $z^{\left(2,\left\|s_{1}, s_{2}+1\right\|\right)}=z^{\left(2,\left\|s_{1}, s_{2}\right\|\right)}+1$ for an arbitrary but fixed $s_{2}$, where $1 \leq s_{2} \leq\left\lfloor\frac{m}{2}\right\rfloor+1$ and $j_{1}=s_{2}+1$. Hence,

$$
z^{\left(2,\left\|s_{1}, s_{2}\right\|\right)}=\operatorname{ini}_{2}+j_{1}-1
$$

where $i n i_{2}$ is the initial value of $z$ before entering the innermost loop. Further, after $s_{2}=j_{1}-1=\left\lfloor\frac{m}{2}\right\rfloor+1$ iterations of the innermost loop, the total number of increments over $z$ is:

$$
\text { incr }_{2}=\sum_{k=1}^{\left\lfloor\frac{m}{2}\right\rfloor+1} 1=\left\lfloor\frac{m}{2}\right\rfloor+1 .
$$

The innermost loop of Figure 4(b) is next eliminated, yielding:

$$
\text { for }\left(i_{1}=1 ; i_{1} \leq n+1 ; i_{1}=i_{1}+1\right) \underline{\text { do }} z=z+\left\lfloor\frac{m}{2}\right\rfloor+1 \text { end do }
$$

with the recurrence equation of $z$ as:

$$
z^{\left(1,\left\|s_{1}\right\|+1\right)}=z^{\left(1,\left\|s_{1}\right\|\right)}+\left\lfloor\frac{m}{2}\right\rfloor+1 .
$$

Solving this recurrence and using that $z_{0}=1$ is the initial value of $z$ before the outermost loop of Figure 4(b), we obtain:

$$
z^{\left(1,\left\|s_{1}\right\|\right)}=1+\sum_{k=1}^{i_{1}-1}\left(\left\lfloor\frac{m}{2}\right\rfloor+1\right)=1+\left(i_{1}-1\right)\left(\left\lfloor\frac{m}{2}\right\rfloor+1\right) .
$$

Computing the $z$-relation among arbitrary values of $z, i_{1}, \ldots, i_{d}$.

We are interested in deriving the value of $z^{\left(d,\left\|s_{1}, \ldots, s_{d}\right\|\right)}$, where $i_{k}=s_{k}+1(k=$ $1, \ldots, d)$, from which the $z$-relation can be immediately constructed as $z=z^{\left(d,\left\|s_{1}, \ldots, s_{d}\right\|\right)}$.

The value $z^{\left(d,\left\|s_{1}, \ldots, s_{d}\right\|\right)}$ (and hence the $z$-relation) is inferred by Algorithm 2 as follows. 
(a) The value incr is computed such that $z^{\left(1,\left\|s_{1}\right\|\right)}=z_{0}+$ incr (line 2 of Algorithm 2);

(b) The outermost loop of (4) is omitted (line 1 of Algorithm 2), yielding:

$$
\begin{aligned}
& \text { for }\left(i_{2}=1 ; i_{2} \leq f_{1}\left(i_{1}\right) ; i_{2}=i_{2}+1\right) \underline{\text { do }} \\
& \quad \ldots \\
& \quad \underline{\text { for }}\left(i_{d}=1 ; i_{d} \leq f_{d-1}\left(i_{1}, \ldots, i_{d-1}\right) ; i_{d}=i_{d}+1\right) \underline{\text { do }} \\
& z:=z+g\left(i_{d}\right) \\
& \quad \underline{\text { end do }}
\end{aligned}
$$

\section{end do}

(c) The value of $z$ at the $s_{2}$ th iteration of the outermost loop (8) is next computed, where the initial value of $z$ before (8) is considered to be $z^{\left(1,\left\|s_{1}\right\|\right)}$ (line 7 of Algorithm 2). As a result, $z^{\left(2,\left\|s_{1}, s_{2}\right\|\right)}$ in the loop (4) is obtained.

(d) Steps (b)-(c) are recursively applied on (8) until no more loops are left and $z^{\left(d,\left\|s_{1}, \ldots, s_{d}\right\|\right)}$ is derived (lines 6-9 of Algorithm 2).

Example 4. Consider Figure 4(b). The outermost loop of Figure 4(b) is omitted (line 1 of Algorithm 2), yielding:

$$
\text { for }\left(j_{1}=1 ; j_{1} \leq\left\lfloor\frac{m}{2}\right\rfloor+1 ; j_{1}=j_{1}+1\right) \underline{\text { do }} z=z+1 \underline{\text { end do }}
$$

The total number of increments incr $_{2}=\left\lfloor\frac{m}{2}\right\rfloor+1$ over $z$ made by (9) is computed, as presented in Example 3 (line 2 of Algorithm 2). The value $z_{i}=z^{\left(1,\left\|s_{1}\right\|\right)}$ of $z$ at an iteration $s_{1}=i_{1}-1$ of the outermost loop of Figure 4(b) is further obtained (lines 3-5 of Algorithm 2), as:

$$
z_{i}=z_{0}+\sum_{n v a r=1}^{i_{1}-1}\left(\left\lfloor\frac{m}{2}\right\rfloor+1\right)=1+\left(i_{1}-1\right)\left(\left\lfloor\frac{m}{2}\right\rfloor+1\right) .
$$

Next, Algorithm 2 is called on (9) with the initial value $z_{i}$ to compute the value of $z$ at an iteration $s_{2}=j_{1}-1$ of (9) (line 7 of Algorithm 2). As (9) has no inner loops, we have $i n c r=1$ and $z_{i}^{\prime}=z_{i}+\sum_{n v a r=1}^{j_{1}-1} 1$, yielding (lines 2-5 of Algorithm 2):

$$
z^{\left(2,\left\|s_{1}, s_{2}\right\|\right)}=z_{i}^{\prime}=\left(i_{1}-1\right)\left(\left\lfloor\frac{m}{2}\right\rfloor+1\right)+j_{1} .
$$

The $z$-relation of Figure 4(b) is finally derived (line 9 of Algorithm 2), as:

$$
z=\left(i_{1}-1\right)\left(\left\lfloor\frac{m}{2}\right\rfloor+1\right)+j_{1} .
$$

To obtain the $z$-relation of Figure 2(b), we make use of $i=i_{1}-1$ and $j=2\left(j_{1}-1\right)$ and have:

$$
z=i\left(\left\lfloor\frac{m}{2}\right\rfloor+1\right)+\left\lfloor\frac{j}{2}\right\rfloor+1 .
$$

Replacing $i$ and $j$ respectively with $n$ and $m$ in the $z$-relation, the upper bound on loop iteration counts of Figure 2(b) is:

$$
(n+1)\left(\left\lfloor\frac{m}{2}\right\rfloor+1\right)
$$




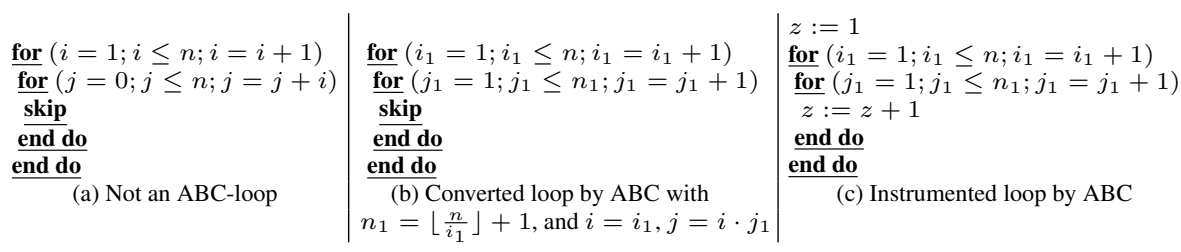

Fig. 5. ABC on a non-ABC-loop.

\subsection{Symbolic Solver}

Simplifying arithmetical expressions and computing closed forms of symbolic sums is performed by the symbolic solver engine of $\mathrm{ABC}$. Our symbolic solver supports the closed form computation of the following sums:

$$
\sum_{x=e_{1}}^{e_{2}} c_{1} \cdot n_{1}^{x} \cdot x^{d_{1}}+\cdots+c_{r} \cdot n_{r}^{x} \cdot x^{d_{r}}
$$

where $e_{1}, e_{2}$ are integer-valued symbolic constants, $n_{i}, d_{i}$ are natural numbers, and $c_{i}$ are rational numbers. Closed forms of such sums always exists [6]. For computing the closed forms of these sums we rely on a simplified version of the Gosper algorithm [6].

We have also instrumented our symbolic solver to handle symbolic sums whose closed forms involve harmonic numbers [7], as discussed in Section 3.5.

\subsection{Beyond ABC-Loops}

Our approach to bound computation implemented in $\mathrm{ABC}$ is complete for $\mathrm{ABC}$-loops and for loops satisfying (2). That is, it always returns the z-relation and loop iteration bound of an ABC-loop.

It is worth to be mentioned that some loops violating (2) can still be successfully analyzed by $\mathrm{ABC}$.

Consider Figure 5(a) violating (2), as updates over $j$ depend on $i$. However, using Algorithm 1 we derive the loop given in Figure 5(b), yielding finally the "instrumented" loop from Figure 5(c). Further, by applying Algorithm 2, we are left with finding the closed form of $\sum_{k=1}^{i_{1}-1}\left\lfloor\frac{n}{i_{1}}\right\rfloor$. This sum cannot be further simplified [7]. However, we can compute an upper-bound on its closed form using the relation:

$$
\sum_{k=1}^{i_{1}-1}\left\lfloor\frac{n}{i_{1}}\right\rfloor \leq\left\lfloor\sum_{k=1}^{i_{1}-1} \frac{n}{i_{1}}\right\rfloor=\left\lfloor n \sum_{k=1}^{i_{1}-1} \frac{1}{i_{1}}\right\rfloor .
$$

Note that $\sum_{k=1}^{i_{1}-1} \frac{1}{i_{1}}$ is the harmonic number $H\left(i_{1}-1\right)$ arising from the truncation of the harmonic series [7]. We make use of $H\left(i_{1}-1\right)$ and derive an upper bound on the loop iteration count of Figure 5(a) as being a harmonic expression. To this end, we have 


\begin{tabular}{|c|c|c|c|}
\hline Loop & $z$-relation & Iteration bound & Time [s] \\
\hline $\begin{array}{l}\text { for }(i=a ; i \leq b ; i=i+1) \\
\text { skip } \\
\text { end do }\end{array}$ & $z=1+i-a$ & $1+b-a$ & 0.172 \\
\hline $\begin{array}{l}\text { for }(i=0 ; i \leq n-1 ; i=i+1) \\
\text { for }(j=0 ; j \leq i ; j=j+1) \\
\text { skip } \\
\text { end do } \\
\text { end do }\end{array}$ & $z=1+j+\frac{i+i^{2}}{2}$ & $\frac{n+n^{2}}{2}$ & 0.219 \\
\hline 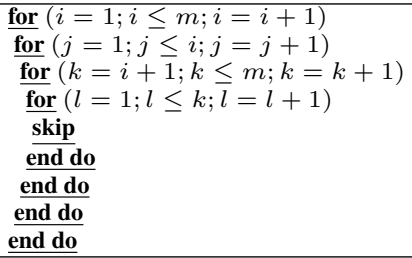 & $\begin{array}{l}z= \\
\frac{i^{2} m^{2}-i m^{2}+i^{2} m-i m}{4}+ \\
\frac{i^{2}-i^{4}}{8}+\frac{i^{3}-i}{12}+ \\
\frac{j m+j m^{2}+k^{2}}{2}- \\
\frac{m^{2}+j i^{2}+j i+m+k}{2}+1\end{array}$ & $\frac{3 m^{4}+2 m^{3}-3 m^{2}-2 m}{24}$ & 1.281 \\
\hline $\begin{array}{l}\text { for }\left(i=0 ; i \leq\left(\frac{n * n * n}{2}-1\right) ; i=i+\right. \\
\text { for }(j=0 ; j \leq n-1 ; j=j+1) \\
\frac{\text { for }(k=0 ; k \leq j-1 ; k=k+1)}{\text { skip }} \\
\text { end do } \\
\underline{\text { end do }} \\
\text { end do }\end{array}$ & $\begin{array}{l}z= \\
1+k+\frac{i n^{2}-i n+j^{2}-j}{2}\end{array}$ & $\frac{n^{5}-n^{4}}{4}$ & 0.234 \\
\hline $\begin{array}{l}\text { for }(i=1 ; i \leq n ; i=i+1) \\
\text { for }(j=1 ; j \leq i ; j=j+1) \\
\text { skip } \\
\text { end do } \\
\text { end do }\end{array}$ & $z=\frac{i^{2}-i}{2}+j$ & $\frac{n^{2}+n}{2}$ & 0.203 \\
\hline $\begin{array}{l}\text { for }(i=1 ; i \leq n ; i=i+1) \\
\text { for }(j=i ; j \leq n ; j=j+1) \\
\text { skip } \\
\text { end do } \\
\text { end do }\end{array}$ & $\begin{array}{l}z= \\
(i-1) n+j+\frac{i-i^{2}}{2}\end{array}$ & $\frac{n^{2}+n}{2}$ & 0.203 \\
\hline $\begin{array}{l}\text { for }(j=1 ; i \leq m ; j=j+1) \\
\text { for }(i=1 ; i \leq n ; i=j+1) \\
\underline{\text { skip }} \\
\underline{\text { end do }}\end{array}$ & $z=i+(j-1) n$ & $n m$ & 0.187 \\
\hline $\begin{array}{l}\text { for }(i=n ; i \geq 1 ; i=i-1) \\
\underline{\text { for }(j=m ; j \geq 1 ; j=j-1)} \\
\underline{\text { skip }} \\
\underline{\text { end do }}\end{array}$ & $z=(n-i+1) m-j+1$ & $n m$ & 0.188 \\
\hline
\end{tabular}

extended our symbolic solver with some simple identities over harmonic numbers. To the best of our knowledge, inferring a tight loop bound for Figure 5(a) is not yet possible by other works.

$\mathrm{ABC}$ can thus be successfully applied to loops for which symbolic computation methods can be deployed to compute or approximate closed forms of loop variables. Such cases may arise from nested loops whose inner loop counters are updated by nonconstant polynomial functions in the outer loop counters (i.e. yielding iteration bounds as harmonic numbers).

\section{Experiments}

We applied $\mathrm{ABC}$ to a large number of examples, including benchmark programs from recent work on timing analysis [21] as well as from the JAMA package [13]. 


\begin{tabular}{|c|c|c|c|}
\hline Loop & $z$-relation & Iteration bound & Time [s] \\
\hline $\begin{array}{l}\text { for }(i=1 ; i \leq n ; i=i+1) \\
\text { for }(j=1 ; j \leq m ; j=j+1) \\
\text { skip } \\
\text { end do } \\
\text { end do }\end{array}$ & $z=j+(i-1) m$ & $n m$ & 0.187 \\
\hline $\begin{array}{l}\text { for }(i=n ; i \geq 1 ; i=i-1) \\
\text { for }(j=1 ; j \leq m ; j=j+1) \\
\text { skip } \\
\text { end do } \\
\text { end do }\end{array}$ & $z=j+(n-i) m$ & $n m$ & 0.187 \\
\hline $\begin{array}{l}\text { for }(i=n ; i \geq 1 ; i=i-1) \\
\text { for }(j=m ; j \geq 1 ; j=j-1) \\
\text { skip } \\
\underline{\text { end do }} \\
\text { end do }\end{array}$ & $z=1-j+(n-i+1) m$ & $n m$ & 0.187 \\
\hline $\begin{array}{l}\text { for }(i=n ; i \geq 1 ; i=i-1) \\
\text { for }(j=m ; j \geq m ; j=j-1) \\
\text { skip } \\
\text { end do } \\
\text { end do }\end{array}$ & $z=1-i-j+m+n$ & $n$ & 0.203 \\
\hline $\begin{array}{l}\text { for }(i=a ; i \leq b ; i=i+1) \\
\text { for }(j=c ; j \leq d ; j=j+1) \\
\frac{\text { for }(k=i-j ; k \leq i+j ; k=k+1)}{\text { skip }} \\
\underline{\text { end do }} \\
\underline{\text { end do }}\end{array}$ & $\begin{array}{l}z= \\
1-2 a d+2 i d- \\
a d^{2}+i d^{2}+a c^{2}- \\
i c^{2}+j^{2}-c^{2}+ \\
j-a+k\end{array}$ & $\left(c^{2}-(d+1)^{2}\right)(a-b-1)$ & 0.328 \\
\hline $\begin{array}{l}\text { for }(i=n ; i \geq 1 ; i=i-1) \\
\text { for }(j=1 ; j \leq m ; j=j+1) \\
\text { for }(k=i ; k \leq i+j ; k=k+1) \\
\frac{\text { for }(l=1 ; l \leq k ; l=l+1)}{\text { skip }} \\
\frac{\underline{\text { end do }}}{\underline{\text { end do }}} \\
\underline{\text { end do }}\end{array}$ & $\begin{array}{l}z= \\
-\frac{m^{2}+3 m+2}{4} i^{2}+ \\
\left(\frac{j^{2}+j-1}{2}-\frac{2 m^{3}+9 m^{2}+13}{12}\right) i+ \\
\frac{k^{2}-k}{2}+\frac{j^{3}-j}{6}+1+ \\
\frac{2 m^{2}+3 m n+9 m+9 n+13}{12} m n\end{array}$ & $\frac{2 m^{2}+3 m n+9 m+9 n+13}{12} m n$ & 0.625 \\
\hline $\begin{array}{l}\text { for }(i=n ; i \geq 1 ; i=i-1) \\
\text { for }(j=1 ; j \leq m ; j=j+1) \\
\text { for }(k=i ; k \leq p ; k=k+1) \\
\underline{\text { for }(l=q ; l \leq j ; l=l-1)} \\
\underline{\text { exip }} \\
\underline{\underline{\text { end do }}} \\
\underline{\underline{\text { end do }}}\end{array}$ & $\begin{array}{l}z= \\
\frac{3 j-i j-j^{2}+i j^{2}}{2}+k-l-p+ \\
\frac{i^{2} m+i m^{2}-i^{2} m^{2}-i m}{4}+ \\
j q-j k+k q-p q+ \\
\frac{m n-m^{2} n-m n^{2}+m^{2} n^{2}}{4}+ \\
\frac{3 j p-j^{2} p-i m p+i m^{2} p}{2}- \\
i j q+j p q+ \\
\frac{m n p-m^{2} n p-i m q}{2}-i m p q+ \\
\frac{i m^{2} q+m n q-m n^{2} q}{2}+m n p q\end{array}$ & $\begin{array}{l}\frac{m^{2} n^{2}-m n^{2}-m^{2} n+m n}{4}+ \\
\frac{m n p-m^{2} n p+m n q-m n^{2} q}{2}+ \\
m n p q\end{array}$ & 0.375 \\
\hline
\end{tabular}

Table 2. Further experimental results obtained by ABC.

Tables 1 and 2 summarize some of our results obtained on a machine with a 2.8 $\mathrm{GHz}$ Intel Core 2 Duo processor and 2GB of RAM.

The first four programs of Table 1 are examples taken from [21], whereas the last four programs of Table 1 are loops taken from the JAMA package [13]. The examples of Table 2 are our own benchmark examples, and illustrate the power of $\mathrm{ABC}$ in handling nested loops whose inner loop counters polynomially depend on its outer loop counters. The difference between the first four programs of Table 2 is given by the mixed incremental/decremental updates and smallest/greatest values of the loop counters. Note that the last three programs of Table 2 yield polynomial loop bounds as sums of multivariate monomials. 
The first column of Table 1 (respectively of Table 2) presents the loop being fed into $\mathrm{ABC}$, the second column shows the $z$-relation derived by $\mathrm{ABC}$, whereas the third one presents the number of loop iterations computed by $\mathrm{ABC}$. The forth column gives the time (in seconds) needed by $\mathrm{ABC}$ to infer bounds on loop iteration counts ${ }^{9}$. Note that iteration bounds are integer-valued polynomial expressions (e.g. $n^{2}+n$ is divisible by 2).

\section{Conclusions}

We describe the software tool $\mathrm{ABC}$ for automatically deriving tight symbolic upper bounds on loop iteration counts of a special class of loops, called the ABC-loops. The system was successfully tried on a large number of examples. The derived symbolic bounds express non-trivial (polynomial and harmonic) relations over loop variables.

Future work includes extending $\mathrm{ABC}$ to handle more complex sums, such as e.g. fractions of polynomials [17], including more sophisticated control-flow refinement techniques, such as [8], into $\mathrm{ABC}$, and improving the loop converter of $\mathrm{ABC}$ for handling more complex loops.

\section{References}

1. E. Albert, P. Arenas, S. Genaim, and G. Puebla. Automatic Inference of Upper Bounds for Recurrence Relations in Cost Analysis. In Proc. of SAS, pages 221-237, 2008.

2. B. Birkeland. Calculus and Algebra with MathCad 2000. Haeftad. Studentlitteratur, 2000.

3. P. Cousot and R. Cousot. Abstract Interpretation: a Unified Lattice Model for Static Analysis of Programs by Construction or Approximation of Fixpoints. In Proc. of POPL, pages 238252, 1977.

4. I. Danaila, P. Joly, S. M. Kaber, and M. Postel. An Introduction to Scientific Computing: Twelve Computational Projects Solved with MATLAB. Springer, 2007.

5. C. Ferdinand and R. Heckmann. aiT: Worst Case Execution Time Prediction by Static Program Analysis. In Proc. of IFIP Congress Topical Sessions, pages 377-384, 2004.

6. R. W. Gosper. Decision Procedures for Indefinite Hypergeometric Summation. PNAS, 75:40-42, 1978.

7. R. L. Graham, D. E. Knuth, and O. Patashnik. Concrete Mathematics. Addison-Wesley Publishing Company, 2nd edition, 1989.

8. S. Gulwani, S. Jain, and E. Koskinen. Control-flow Refinement and Progress Invariants for Bound Analysis. In Proc. of PLDI, pages 375-385, 2009.

9. J. Gustafsson, A. Ermedahl, C. Sandberg, and B. Lisper. Automatic Derivation of Loop Bounds and Infeasible Paths for WCET Analysis Using Abstract Execution. In Proc. of RTSS, pages 57-66, 2006.

10. C. A. Healy, M. Sjödin, V. Rustagi, D. B. Whalley, and R. van Engelen. Supporting Timing Analysis by Automatic Bounding of Loop Iterations. Real-Time Systems, 18(2/3):129-156, 2000.

11. T. A. Henzinger, T. Hottelier, and L. Kovacs. Valigator: A Verification Tool with Bound and Invariant Generation. In Proc. of LPAR, pages 333-342, 2008.

12. M. V. Hermenegildo, G. Puebla, F. Bueno, and P. Lopez-Garcia. Integrated Program Debugging, Verification, and Optimization using Abstract Interpretation (and the Ciao System Preprocessor). Sci. Comput. Program., 58(1-2):115-140, 2005.

\footnotetext{
${ }^{9}$ Note, that the timings given in Tables 1 and 2 include also the required time to launch the JVM.
} 
13. J. Hicklin, C. Moler, P. Webb, R. F. Boisvert, B. Miller, R. Pozo, and K. Remington. JAMA: A Java Matrix Package. http://math.nist.gov/javanumerics/jama/, 2005.

14. S. Jost, H. Loidl, K. Hammond, N. Scaife, and M. Hofmann. "Carbon Credits" for ResourceBounded Computations Using Amortised Analysis. In Proc. of FM, pages 354-369, 2009.

15. M. Müller-Olm, M. Petter, and H. Seidl. Interprocedurally Analyzing Polynomial Identities. In Proc. of STACS, pages 50-67, 2006.

16. J. Navas, E. Mera, P. Lopez-Garcia, and M. V. Hermenegildo. User-Definable Resource Bounds Analysis for Logic Programs. In Proc. of ICLP, pages 348-363, 2007.

17. I. Nemes and M. Petkovsek. RComp: A Mathematica Package for Computing with Recursive Sequences. Journal of Symbolic Computation, 20(5-6):745-753, 1995.

18. M. Odersky. The Scala Language Specification. http: / / www . scala-lang . org, 2008.

19. A. Prantl, J. Knoop, M. Schordan, and M. Triska. Constraint Solving for High-Level WCET Analysis. CoRR, abs/0903.2251, 2009.

20. G. W. Stewart. JAMPACK: A Java Package For Matrix Computations. http://www.mathematik.hu-berlin.de/ lamour/software/JAVA/Jampack/.

21. R. A. van Engelen, J. Birch, and K. A. Gallivan. Array Data Dependence Testing with the Chains of Recurrences Algebra. In Proc. of IWIA, pages 70-81, 2004.

22. S. Wolfram. The Mathematica Book. Version 5.0. Wolfram Media, 2003. 